\title{
ELEMENTS OF PRIME POWER ORDER AND THEIR CONJUGACY CLASSES IN FINITE GROUPS
}

\author{
LÁSZLÓ HÉTHELYI and BURKHARD KÜLSHAMMER
}

(Received 6 May 2003; revised 17 December 2003)

Communicated by E. A. O'Brien

\begin{abstract}
We show that, for any positive integer $k$, there are only finitely many finite groups, up to isomorphism, with exactly $k$ conjugacy classes of elements of prime power order. This generalizes a result of $E$. Landau from 1903. The proof of our generalization makes use of the classification of finite simple groups.
\end{abstract}

2000 Mathematics subject classification: primary $20 \mathrm{C} 45$.

\section{Introduction}

Landau has proved that, for any positive integer $k$, there are only finitely many finite groups, up to isomorphism, with exactly $k$ conjugacy classes [3]. In this paper we prove a variant of Landau's result in which we restrict our attention to conjugacy classes of elements of prime power order only.

THEOREM 1.1. For any positive integer $k$, there are only finitely many finite groups, up to isomorphism, with exactly $k$ conjugacy classes of elements of prime power order.

Whereas the proof of Landau's original result is elementary, our proof of Theorem 1.1 relies on the classification of finite simple groups. Theorem 1.1 is also related to a conjecture of Praeger [4, page 30]. We are grateful to L. Pyber for pointing out this reference.

In the following, we denote by $\operatorname{kpp}(G)$ the number of conjugacy classes of elements of prime power order in a finite group $G$. (Throughout the conjugacy class of 1 is counted as one of the conjugacy classes of elements of prime power order.)

(C) 2005 Australian Mathematical Society $1446-7887 / 05 \$ A 2.00+0.00$ 
LEMMA 1.2. Let $N$ be a normal subgroup of a finite group $G$. Then

(i) $\operatorname{kpp}(G) \leq \operatorname{kpp}(G / N) \cdot|N|$;

(ii) $\operatorname{kpp}(G / N)<\operatorname{kpp}(G)$ unless $N=1$.

PROOF. Let $N$ be arbitrary, and let $C$ be a conjugacy class of elements of prime power order in $G$. Then the image $\bar{C}$ of $C$ in $\bar{G}=G / N$ is a conjugacy class of elements of prime power order in $\bar{G}$.

Conversely, let $x N$ be an element in $G / N$ whose order is a power $p^{n}$ of a prime $p$. We write $x=x_{p} x_{p^{\prime}}=x_{p^{\prime}} x_{p}$ where $x_{p}$ is a $p$-element and $x_{p^{\prime}}$ is a $p^{\prime}$-element in $G$. Then $x N=\left(x_{p} N\right)\left(x_{p^{\prime}} N\right)=\left(x_{p^{\prime}} N\right)\left(x_{p} N\right)$ where $x_{p} N$ is a $p$-element and $x_{p^{\prime}} N$ is a $p^{\prime}$-element. Since $x N$ has order $p^{n}$, we must have $x_{p^{\prime}} N=1$. Thus $x N=x_{p} N$, and we see that $C \mapsto \bar{C}$ is a map from the set of conjugacy classes of elements of prime power order in $G$ onto the set of conjugacy classes of elements of prime power order in $\bar{G}=G / N$.

Let $N \neq 1$. Then $N$ contains an element $x \neq 1$ of prime order. Thus the conjugacy classes of $x$ and 1 have the same image in $G / N$. Hence $\operatorname{kpp}(G / N)<\operatorname{kpp}(G)$.

Now let $\bar{C}$ be a conjugacy class of elements of prime power order in $\bar{G}$. Then the pre-image of $\bar{C}$ in $G$ consists of $|\bar{C}| \cdot|N|$ elements. These form a union of conjugacy classes $C_{1}, \ldots, C_{r}$ of $G$. For $i=1, \ldots, r$, we have $\overline{C_{i}}=\bar{C}$ and hence $\left|C_{i}\right| \geq\left|\overline{C_{i}}\right|=|\bar{C}|$. Hence $r \leq|N|$, and the result is proved.

We are now going to prove Theorem 1.1 in a series of lemmas.

LEMMA 1.3. There exists a function $\alpha: \mathbb{N} \rightarrow \mathbb{N}$ with the following property: Whenever $k$ is a positive integer and $G$ is a finite simple group with $\operatorname{kpp}(G)=k$ then $|G| \leq \alpha(k)$.

Proof. Let $k \in \mathbb{N}$, and let $G$ be a finite simple group with $\operatorname{kpp}(G)=k$. We wish to show that $|G|$ is bounded in terms of $k$. (Our proof will make use of the classification of finite simple groups.) Our claim is trivial if $G$ has prime order, or if $G$ is a sporadic simple group. If $G$ is an alternating group $A_{n}$ then $|G|=n ! / 2$ can have at most $k$ different prime divisors, so $|G|$ is also bounded in this case.

Thus, in the remainder of the proof, we may assume that $G$ is a finite simple group of Lie type. There are 16 such families of groups (see [1, page 8]). It suffices to show that there are only finitely many possibilities for $G$ in each family.

Suppose first that $G=\operatorname{PSL}(n, q)$ for some $n>1$ and some prime power $q$, so that

$$
|G|=(n, q-1)^{-1} q^{\left(\begin{array}{l}
n \\
2
\end{array}\right)}\left(q^{n}-1\right) \cdots(q-1) .
$$

The Zsigmondy prime number theorem (see [2, IX.8.3]) shows that every factor $q^{i}-1$ of $|G|$ with $i>6$ contributes a new prime divisor of $|G|$ and thus a new conjugacy 
class of elements of prime (power) order. Hence $k=\operatorname{kpp}(G) \geq n-6$, and we have shown that $n$ is bounded in terms of $k$, in case of $G=\operatorname{PSL}(n, q)$.

We now keep $n$ fixed and show that $q$ is also bounded in terms of $k$. Let $\widehat{G}:=$ $\operatorname{SL}(n, q)$ and $\widehat{Z}:=Z(\widehat{G})$, so that $|\widehat{Z}|=(n, q-1)$. We keep $|\widehat{Z}|$ fixed. Now $\widehat{G}$ contains a maximal torus $\widehat{T}$ of order $(q-1)^{n-1}$. We write the prime factorization of $|\widehat{T}|$ in the form $|\widehat{T}|=p_{1}^{a_{1}} \cdots p_{m}^{a_{m}}$. Then again $m$ is bounded in terms of $k$. We regard $m$ as fixed. Then $\widehat{T}$ contains $p_{1}^{a_{1}}+\cdots+p_{m}^{a_{m}}-m+1$ elements of prime power order.

Let $\mathbf{F}$ denote the algebraic closure of the finite field $\mathbf{F}_{q}$ with $q$ elements. The elements of $\widehat{T}$ can be diagonalized simultaneously in $\operatorname{GL}(n, \mathbf{F})$. Two diagonal matrices in $\operatorname{GL}(n, \mathbf{F})$ are conjugate if and only if one can be obtained from the other by permuting the diagonal entries. Hence our $p_{1}^{a_{1}}+\cdots+p_{m}^{a_{m}}-m+1$ elements fall into at least $(n !)^{-1}\left(p_{1}^{a_{1}}+\cdots+p_{m}^{a_{m}}-m+1\right)$ different conjugacy classes under $\operatorname{GL}(n, \mathbf{F})$. Thus

$$
\operatorname{kpp}(\widehat{G}) \geq(n !)^{-1}\left(p_{1}^{a_{1}}+\cdots+p_{m}^{a_{m}}-m+1\right),
$$

and Lemma 1.2 implies that

$$
k=\operatorname{kpp}(G) \geq \operatorname{kpp}(\widehat{G}) /|\widehat{Z}| \geq(n, q-1)^{-1}(n !)^{-1}\left(p_{1}^{a_{1}}+\cdots+p_{m}^{a_{m}}-m+1\right) .
$$

Hence $p_{1}^{a_{1}}, \ldots, p_{m}^{a_{m}}$ are bounded in terms of $k$; in particular, $(q-1)^{n-1}=p_{1}^{a_{1}} \cdots p_{m}^{a_{m}}$ is bounded in terms of $k$. Thus certainly $q$ is bounded in terms of $k$. This finishes the proof in case $G=\operatorname{PSL}(n, q)$.

The argument is similar for the other families of finite simple groups of Lie type, and will therefore be omitted. This finishes the proof of Lemma 1.3.

LEMMA 1.4. There exists a function $\beta: \mathbb{N} \rightarrow \mathbb{N}$ with the following property: Whenever $k$ is a positive integer and $G$ is a characteristically simple finite group with $\operatorname{kpp}(G)=k$ then $|G| \leq \beta(k)$.

PROOF. Let $k$ be a positive integer, and let $G$ be a characteristically simple finite group with $\operatorname{kpp}(G)=k$. We know that $G \cong S^{r}=S \times \cdots \times S$ ( $r$ factors) for a finite simple group $S$ and a positive integer $r$. Now certainly $\operatorname{kpp}(G) \geq r(\operatorname{kpp}(S)-1)$. Thus $r \leq k$ and $\operatorname{kpp}(S) \leq k$. By Lemma 1.3, we have

$$
|S| \leq \max \{\alpha(1), \ldots, \alpha(k)\}=: A(k) .
$$

Hence $|G| \leq A(k)^{k}=: \beta(k)$, and the Lemma is proved.

The following Lemma implies Theorem 1.1.

LEMMA 1.5. There exists a function $\gamma: \mathbb{N} \rightarrow \mathbb{N}$ with the following property: Whenever $k$ is a positive integer and $G$ is a finite group with $\operatorname{kpp}(G)=k$ then $|G| \leq \gamma(k)$. 
ProOF. We define $\gamma(k)$ inductively, starting with $\gamma(1):=1$. Then the result is certainly true for $k=1$. So let us assume that $k>1$, and that $\gamma(1), \ldots, \gamma(k-1)$ have been defined already. Moreover, let $G$ be a finite group with $\operatorname{kpp}(G)=k$, and let $N$ be a minimal normal subgroup of $G$. Then $\operatorname{kpp}(G / N)<k$ by Lemma 1.2 (ii) since $N \neq 1$, so that

$$
|G / N| \leq \max \{\gamma(1), \ldots, \gamma(k-1)\}=: \Gamma(k-1),
$$

by induction. Also, $N$ contains at most $k G$-conjugacy classes of elements of prime power order. Each of these splits into at most $|G: N| N$-conjugacy classes of elements of prime power order. Thus $N$ contains at most $k \Gamma(k-1)$ conjugacy classes of elements of prime power order. Since $N$ is characteristically simple we conclude that

$$
|N| \leq \max \{\beta(i): i=1, \ldots, k \Gamma(k-1)\}=: B(k) .
$$

Thus $|G| \leq B(k) \Gamma(k-1)=: \gamma(k)$, and our result is proved.

Our proof of Theorem 1.1 is now complete. At the end of this paper, we will discuss some related questions. Let $\pi$ be a set of primes, and let $\pi^{\prime}$ denote the set of primes not contained in $\pi$. In the following, $\mathrm{k}_{\pi}(G)$ is defined as the number of conjugacy classes of $\pi$-elements in a finite group $G$, and $\mathrm{k}_{\pi^{\prime}}(G)$ is defined in a similar way.

(1) Suppose that $A$ is a $\pi$-group, that $B$ is a $\pi^{\prime}$-group, and that $G=A \times B$ is their direct product. Then $\mathrm{k}(G)$, the number of conjugacy classes of $G$, satisfies

$$
\mathrm{k}(G)=\mathrm{k}(A) \mathrm{k}(B)=\mathrm{k}_{\pi}(G) \mathrm{k}_{\pi^{\prime}}(G) .
$$

One may ask whether the inequality

$$
\mathrm{k}(G) \leq \mathrm{k}_{\pi}(G) \mathrm{k}_{\pi^{\prime}}(G)
$$

holds for an arbitrary finite group $G$. This, however, is not the case: Let $\pi=\{3\}$, and let $G$ be a dihedral group of order $6 q$ where $q$ is a prime different from 2 and 3 . Then we have

$$
\mathrm{k}(G)=(3 q+3) / 2, \quad \mathrm{k}_{\pi}(G)=2, \quad \mathrm{k}_{\pi^{\prime}}(G)=\mathrm{k}(G / P)=(q+3) / 2,
$$

with $P:=O_{3}(G)$. Thus

$$
\mathrm{k}_{\pi}(G) \mathrm{k}_{\pi^{\prime}}(G)=q+3<(3 q+3) / 2=\mathrm{k}(G) .
$$

(2) Now let $A$ be a finite $\pi^{\prime}$-group acting faithfully on a finite $\pi$-group $B$, and let $G$ be the corresponding semidirect product. One may ask whether

$$
\mathrm{k}_{\pi}(G) \mathrm{k}_{\pi^{\prime}}(G) \leq|B| .
$$


However, this is not true, in general. For example, let $\pi=\{p\}$ for an odd prime number $p$, and let $G=\operatorname{AGL}(1, p)$ be the affine general linear group of degree 1 over the field with $p$ elements. Then $G$ is the semidirect product of a cyclic group $A$ of order $p-1$ and a cyclic group $B$ of order $p$. Moreover, we have $\mathrm{k}_{\pi}(G)=2$ and $\mathrm{k}_{\pi^{\prime}}(G)=p-1$, but

$$
|B|=p<2(p-1)=\mathrm{k}_{\pi}(G) \mathrm{k}_{\pi^{\prime}}(G)
$$

\section{Acknowledgements}

The authors gratefully acknowledge financial support from the program 'Algorithmic investigations of discrete algebraic structures' sponsored by the BMBF and the TeT foundation (D-4/99). The first named author was also supported by OTKA grants T034878 and T042481. Both authors are grateful to the referee for a number of helpful suggestions.

\section{References}

[1] D. Gorenstein, R. Lyons and R. Solomon, The classification of the finite simple groups I (Amer. Math. Soc., Providence, 1994).

[2] B. Huppert and N. Blackburn, Finite groups II (Springer, Berlin, 1982).

[3] E. Landau, 'Über die Klassenzahl der binären quadratischen Formen von negativer Diskriminante', Math. Ann. 56 (1903), 671-676.

[4] C. E. Praeger, 'Kronecker classes of fields and covering subgroups of finite groups', J. Austral. Math. Soc. (Series A) 57 (1994), 17-34.

Department of Algebra

Technical University of Budapest

H-1521 Budapest

Hungary

e-mail: hethelyi@math.bme.hu
Mathematical Institute University of Jena D-07737 Jena

Germany e-mail: kuelshammer@uni-jena.de 